Objective To investigate the use of hamstring muscle strengthening in female elite football training.

Design Descriptive, cross-sectional study.

Setting Online questionnaire.

Participants Overall, 338 Danish players were invited to answer the online questionnaire. 123 players from the best national league answered the questionnaire ( $\leq 18$ yrs.: $N=70$ ) and ( $\geq 18$ yrs: $N=53$ ).

Assessment of Risk Factors The online questionnaire covered e. g. baseline information, years of football experience, previous injuries and amount of hours pr/week doing strength training. The following question was asked: 'Which of the following exercises are a part of your weekly training?' With the following options: Leg Curl, Deadlift, Nordic Hamstring and Kettlebell Swing.

Main Outcome Measurements Four hamstring strength exercises: Leg Curl, Deadlift, Nordic Hamstring and Kettlebell Swing.

Results $54,7 \%$ of the adult players reported that they did strength training 3-4 hours/week vs. $39,1 \%$ of the youth elite players. All four hamstrings exercises were reported to be a part of the weekly training in both groups: The Nordic Hamstring exercise was performed among $75 \%$ of the adult elite players vs $70 \%$ of the youth elite players. Deadlift $77 \%$ (adult) vs $53 \%$ (youth). Kettlebell swing 36\% (adult) vs 44\% (youth) and finally the Leg curl 40\% (adult) vs. $14 \%$ (youth)

Conclusions The present results indicate that hamstrings exercises are implemented in the weekly training among the majority of Danish female elite football players. The Nordic Hamstring is overall the most performed hamstring exercise in both groups. However, it remains to be elucidated how the exercise is implemented in terms of frequency and load.

\section{PREDICTORS OF TIME TO RETURN TO PLAY AND REINJURY FOLLOWING HAMSTRING STRAIN INJURIES WITH AND WITHOUT TENDON INVOLVEMENT IN PROFESSIONAL FOOTBALL}

${ }^{1}$ Scott McAuley, ${ }^{2}$ Nick Dobbin, ${ }^{2}$ Peter Goodwin, ${ }^{1}$ Chris Morgan. 'Liverpool FC, Liverpool, UK; ${ }^{2}$ Department of Health Professions, Manchester Metropolitan University, Manchester, $U K$

\subsection{6/bjsports-2021-IOC.145}

Background Hamstring strain injury (HSI) account for $~ 12 \%$ of all injuries in professional football. It is suggested HSIs extending into the intramuscular tendon are more prone to reinjury, resulting in greater time-loss and delayed time to return to play (RTP). Currently, there is a lack of evidence regarding the effects of HSI with and without intramuscular tendon involvement as well as the impact of factors such as removal of a player and grade.

Objectives 1) Describe the number of HSIs using the British Athletics Muscle Injury Classification (BAMIC); 2) Determine if intramuscular tendon HSI results in extended RTP and higher reinjury risk; 3) Determine the predictors of RTP and reinjury.

Methods A retrospective study in one English Premier League football club over four seasons. Players included underwent an MRI within 7 days of injury and were graded by a
Radiologist using the BAMIC (0a-4). RTP and reinjury rates were recorded along with information on whether the players was removed from play. Data were analysed using a KruskalWallis test and linear regression.

Results Twenty-nine HSI across 24 players (age $=26 \pm 4$ years) were recorded over 4 seasons. There was a significant difference in RTP between grades $1 \mathrm{a}$ and $2 \mathrm{c}(P=0.027)$. No significant difference was observed in RTP between $2 b$ and $2 c$ and no greater risk of reinjury. Grade of HSI $(P=<0.000)$ and removal of the player $(P=0.001)$ were significant predictors of RTP. An increase in grade of HSI resulted in an additional 4 days RTP and removal from the field of play $(P$ $=0.001)$ resulted in an additional 10 days.

Conclusion HSIs extending into the intramuscular tendon (2b cf. 2c) do not influence RTP or reinjury, however RTP is affected by the removal of a player and overall grade.

\section{STRENGTH ENDURANCE AND FATIGABILITY: CANDIDATE RISK FACTORS FOR HAMSTRING RE-INJURY IN SPRINT SPORTS?}

${ }^{2}$ Kathelijn Polspoel, ${ }^{1} J o s$ Vanrenterghem, ${ }^{2}$ Stijn Bogaerts. ${ }^{1} \mathrm{KU}$ Leuven, Leuven, Belgium; ${ }^{2} \mathrm{UZ}$ Leuven, Leuven, Belgium

\subsection{6/bjsports-2021-IOC.146}

Background Hamstring strain injuries (HSI) are characterized by a very high recurrence rate. Despite indications from the field that deficits in strength endurance and fatigability play a role in (re-)injury, screening for these in Return To Sport (RTS) decision making is still not common and requires further investigation.

Objective To justify hamstring strength endurance and fatigability at time of RTS as potential risk factors for re-injury.

Design Observational.

Setting Academic sports rehabilitation centre.

Patients (or Participants) 13 recently injured HSI athletes (M/ F;18-35yr) competitive in sprint sports, at time of RTS, and 17 healthy population-matched controls.

Interventions (or Assessment of Risk Factors) All participants underwent a RTS test battery including clinical and functional tests. For this study, single leg hamstring bridge endurance test (SLHB) and maximal isometric hamstring strength test (MIHS) in inner, mid and outer range, using hand-held dynamometry, were investigated. MIHS was retested after a shuttle-run until exhaustion fatigue protocol. Differences between injured (INJ) and uninjured (UNINJ) limb, and differences between injured and healthy athletes (CON) were tested using Students' t-tests and mixed model ANOVA.

Main Outcome Measurements SLHB [number of repetitions]; MIHS [Newton].

Results Concerning SLHB, injured athletes showed a clinically relevant deficit in hamstring strength endurance compared to their healthy peers, both in their injured and un-injured leg $(\mathrm{INJ}=19.0 \pm 5.0 ; \quad \mathrm{UNINJ}=18.9 \pm 5.4 ; \quad \mathrm{CON}=24.5 \pm 5.3$; $\mathrm{t}=2.6 \& 2.7 ; \mathrm{p}=0.01 \& 0.01$ respectively). For MIHS, a main group effect was found for outer range (INJ=347.5 \pm 67.3 ; $\mathrm{CON}=394.8 \pm 63.8 ; \mathrm{F}=6.1 ; \mathrm{p}=0.02)$, revealing that isometric strength was significantly worse in the injured leg compared to healthy controls. An overall fatigue effect was found $(\mathrm{F}=7.8 ; \mathrm{p}=0.01)$, adding to the injured athletes' baseline deficit in MIHS performance. 